ARTICLE

https://doi.org/10.1038/s41467-019-09531-0

\title{
Beating the exclusion rule against the coexistence of robust luminescence and ferromagnetism in chalcogenide monolayers
}

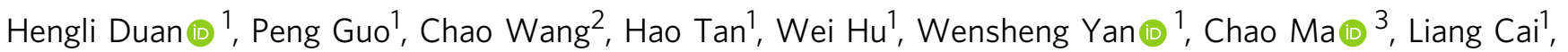
Li Song (1) ${ }^{1}$, Wenhua Zhang ${ }^{1}$, Zhihu Sun (1) ${ }^{1}$, Linjun Wang ${ }^{4}$, Wenbo Zhao ${ }^{5}$, Yuewei Yin (D) ${ }^{5}$, Xiaoguang Li (D) ${ }^{5} \&$ Shiqiang Wei(i) ${ }^{1}$

Monolayer chalcogenide semiconductors with both luminescent and ferromagnetic properties are dreamed for simultaneous polarization and detection of the valley degree of freedom in valleytronics. However, a conventional chalcogenide monolayer lacks these coexisting properties due to their mutually exclusive origins. Herein we demonstrate that robust ferromagnetism and photoluminescence $(\mathrm{PL})$ could be achieved in a ( $\mathrm{Co}, \mathrm{Cr}$ )-incorporated single monolayer $\mathrm{MoS}_{2}$, where the ferromagnetic interaction is activated by $\mathrm{Co}$ ions, and the nonradiative recombination channels of excitons is cut off by $\mathrm{Cr}$ ions. This strategy brings a 90-fold enhancement of saturation magnetization and 35-fold enhancement of PL intensity than the pristine $\mathrm{MoS}_{2}$ monolayer. The main reasons for the coexisting ferromagnetism and $\mathrm{PL}$ are the electronic interactions between the impurity bands of atop $\mathrm{Cr}$ adatoms and substitutional Co atoms, as well as the increased content of neutral exciton. Our findings could extend the applications of two-dimensional chalcogenides into spintronics, valleytronic and photoelectric devices.

\footnotetext{
${ }^{1}$ National Synchrotron Radiation Laboratory, University of Science and Technology of China, 230029 Hefei, Anhui, China. ${ }^{2}$ Key Laboratory of Neutronics and Radiation Safety, Institute of Nuclear Energy Safety Technology, Chinese Academy of Sciences, 230031 Hefei, Anhui, China. ${ }^{3}$ College of Materials Science and Engineering, Hunan University, 410082 Changsha, Hunan, China. ${ }^{4}$ Center for Micro- and Nanoscale Research and Fabrication, University of Science and Technology of China, 230029 Hefei, Anhui, China. ${ }^{5}$ Hefei National Laboratary for Physical Sciences at the Microscale, Department of Physics, and CAS Key Laboratory of Strongly-coupled Quantum Matter Physics, University of Science and Technology of China, 230026 Hefei, Anhui, China. These authors contributed equally: Hengli Duan, Peng Guo. Correspondence and requests for materials should be addressed to W.Y. (email: ywsh2000@ustc.edu.cn) or to Z.S. (email: zhsun@ustc.edu.cn) or to S.W. (email: sqwei@ustc.edu.cn)
} 
M onolayer transition-metal dichalcogenides (TMDCs) with unit-cell thickness have emerged as a frontier for the exploration of physics and next-generation valleytronic devices, owing to the presence of an additional valley degree of freedom (DOF) that is strongly coupled with $\operatorname{spin}^{1-6}$. Effective manipulation and detection of the valley DOF are key issues for practical applications of monolayer TMDCs in valleytronics ${ }^{1,7-10}$. Currently, manipulation of the valley DOF is mainly performed under external fields such as optical, magnetic, and electrical fields. However, a very high magnetic field (beyond several Tesla) is usually required because of the non-magnetic nature of monolayer TMDCs ${ }^{11-13}$. When an external electric field is applied to manipulate the valley DOF, the spin-valley coupling makes it necessary to use an extra magnetic semiconductor in order to inject spin-polarized charge carriers into the nonmagnetic TMDCs; this makes the valleytronic devices very complicated $^{14}$. Besides, the efficiency of electric control of the valley DOF is severely limited by the interface between the magnetic semiconductor and TMDCs. On the other hand, detection of the polarization of the valley could be achieved by virtue of the chirality of the photoluminescence (PL) emitted by TMDCs ${ }^{15,16}$. Unfortunately, the optical detection is hampered by the low PL quantum yield of TMDCs obtained so far. In order to fabricate highly integrated valleytronics devices with the long dreamed capability of storing and processing information at the same time, simultaneous polarization and detection of the valley DOF would provide an opportunity. Toward this goal, monolayer TMDCs with both robust room-temperature ferromagnetism (FM) and PL will play a critical fundamental role and are highly desired.

Among a variety of TMDCs, monolayer $\mathrm{MoS}_{2}$ is a typical material and has attracted enormous attention due to their outstanding mechanical, optical, magnetic, and electronic properties that render them numerous potential applications. Tremendous efforts have been attempted and great progresses have been made in separately exploring and promoting the optical and ferromagnetic properties of monolayer $\mathrm{MoS}_{2}{ }^{17-21}$. For instance, Amani and co-workers enhanced the PL quantum yield of monolayers $\mathrm{MoS}_{2} 100$ times by using a surface passivation strategy to eliminate sulphur vacancy-mediated nonradiative recombination ${ }^{22}$. Chemical doping of $p$-type impurities in $\mathrm{MoS}_{2}$ monolayer could also increase the PL intensity as a result of the reduced concentration of electron carriers ${ }^{18}$. Attempt to tune the PL intensity has also been made by Zhang et al. ${ }^{19}$ through doping the magnetic $\mathrm{Mn}$ ions in CVD-prepared $\mathrm{MoS}_{2}$ monolayer; unfortunately, the PL is actually quenched due to the formation of nonradiative recombination. Inferred from these results, high PL intensity could only be obtained in TMDCs deficient in magnetic ions and sulphur vacancy. However, the presence of abundant magnetic ions and sulphur vacancies are essential prerequisites for achieving $\mathrm{FM}$ of $\mathrm{MoS}_{2}$, as reported by Yan et al. ${ }^{20}$ and Andriotis et al. ${ }^{21}$. A dilemma then presents itself immediately. On the one hand, magnetic TM impurities or sulphur vacancy is indispensable to FM. But on the other hand, magnetic ions and sulphur vacancy tend to form defect-mediated nonradiative recombination and charged exciton (trion), both of which are detrimental to the luminescence. In other words, in analogy to the nonexistence of FM in a superconductor, the presence of FM and PL in the monolayer $\mathrm{MoS}_{2}$ is mutually exclusive: ferromagnetic ordering relies on the magnetic dopants and/or sulphur vacancy, which however quench the PL.

The dilemma above might be solved through the synergetic incorporation of two types of TMs with distinct natures. The first magnetic TM element is employed to induce the spin-polarized bandgap impurity. In many mono-doping cases, the induced impurity band with high state density is localized near the bottom of the conduction band (CB). The excited photoelectrons are apt to hop into this dense band, which decreases the probability of radiative recombination between electrons in the $\mathrm{CB}$ and holes in the valence band $(\mathrm{VB})$ : this is why the mono-doping is detrimental to the PL ${ }^{23}$. Provided that a second TM element capable of reducing the state density of this impurity band is incorporated, the nonradiative channel of the photo-excited electrons could be cut off and hence the radiative recombination probability could be enhanced (Fig. 1). Two delicately chosen $3 d$ TM elements could be expected to meet this requirement, because their $d-d$ electronic interactions allow for the redistribution of their impurity bands as observed in many co-doping systems ${ }^{24,25}$. Furthermore, as the reaction reactivity of TM elements are relevant to the electron filling of their $3 d$ orbitals (below or above half-filling), TM elements of different reactivity tend to occupy different spatial positions in the chalcogenide host during the preparations of the monolayer specimen ${ }^{26,27}$. Therefore, there are a diversity of choices of incorporated TM elements to mediate their $d-d$ synergetic interactions and to tailor the band structure of the monolayers, providing a flexible platform for tuning the luminescent and magnetic properties of these materials.

In this work, we propose a practical approach to bring remarkable PL and FM to TMDCs, by using two types of alien TM elements which play distinct roles in introducing magnetic moments and suppressing the nonradiative recombination of excitons. Experimentally, taking $\mathrm{MoS}_{2}$ monolayers as a prototype material and using the CVD method, we successfully observed both luminescent and room-temperature FM in ( $\mathrm{Co}, \mathrm{Cr})$-incorporated $\mathrm{MoS}_{2}$ monolayers. Detailed characterizations of the structure-property correlations unravel that the substitutional Co atoms induce a bandgap impurity band that gives rise to the ferromagnetic ordering but reduces the PL intensity because of the nonradiative recombination of excitons. After incorporating $\mathrm{Cr}$ atoms standing on atop sites of the monolayer, the nonradiative recombination is suppressed by the electronic interactions between the $\mathrm{Cr}-$ and Co-induced impurity levels. Consequently, the (Co, Cr) $-\mathrm{MoS}_{2}$ monolayers exhibit a 90 -fold

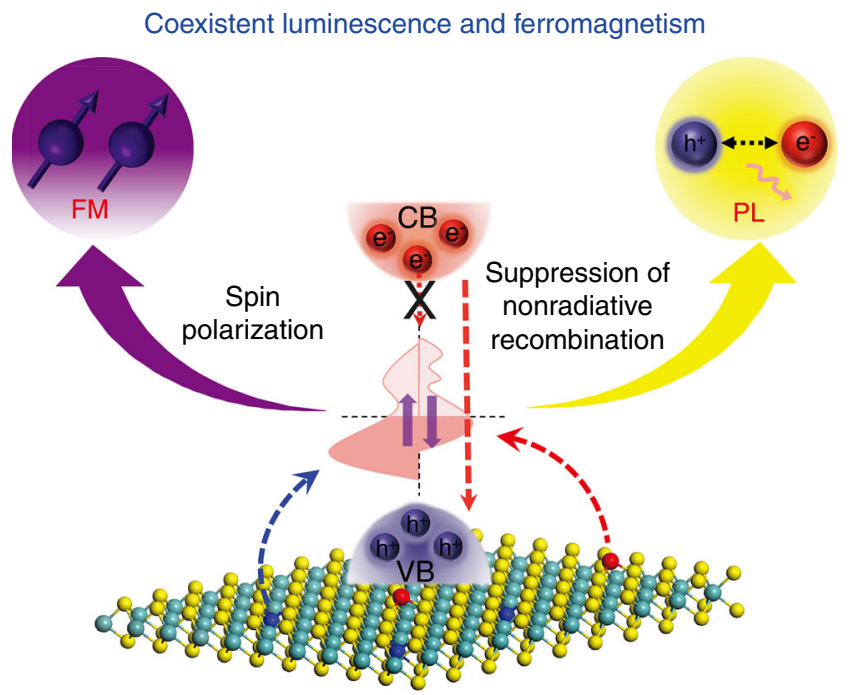

Band-structure engineering

Fig. 1 Diagrammatic representation of the coexistence principle of photoluminescence and ferromagnetism. A possible way to bring the coexistent photoluminescence and ferromagnetism to monolayer TMDCs, through a band-structure engineering that retains the spin polarization of the impurity bands while suppresses the nonradiative recombination of excitons 
a
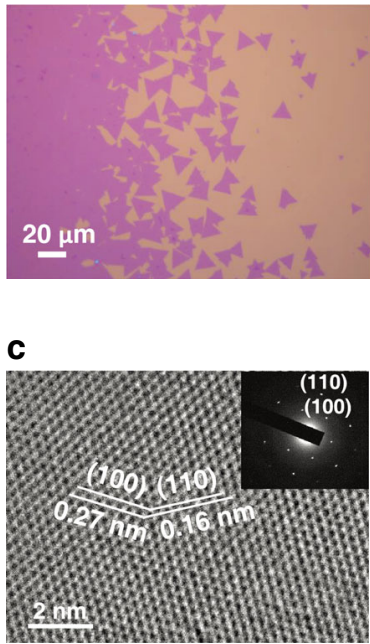

b

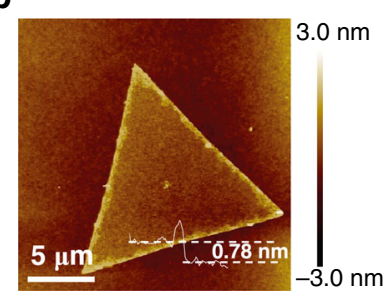

d

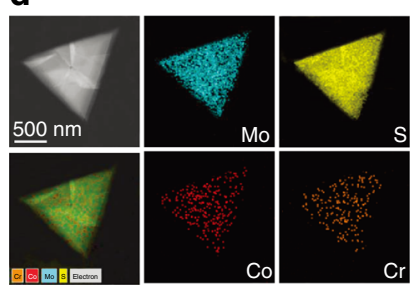

e

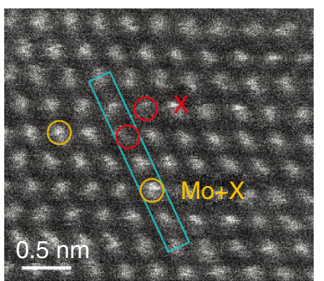

g

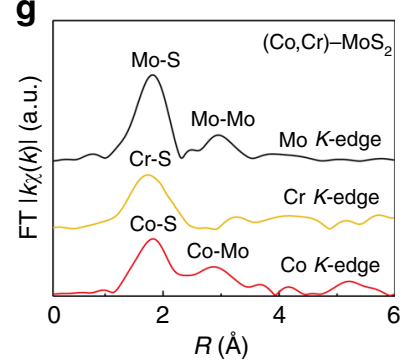

f

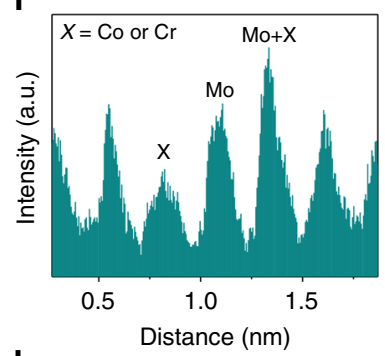

h

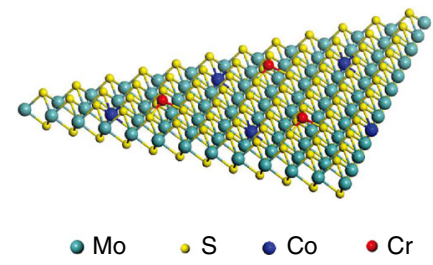

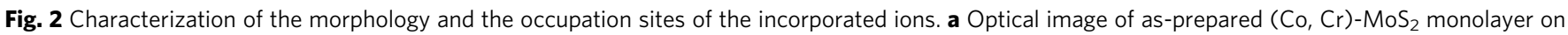
$300 \mathrm{~nm} \mathrm{SiO}_{2} / \mathrm{Si}$ substrates prepared by CVD. b AFM image, c HRTEM image, d EDX elemental mapping images, e HAADF-STEM image, and f the intensity spectra of the selected area of ( $\mathrm{Co}, \mathrm{Cr}$ )-MoS $\mathrm{M}_{2}$ monolayer. $\mathbf{g}$ The FT curves of the Co, $\mathrm{Cr} \mathrm{K}$-edge EXAFS $k \chi(k)$ functions for ( $\left.\mathrm{Co}, \mathrm{Cr}\right)-\mathrm{MoS}{ }_{2}$ monolayer. The Mo K-edge FT curve for $\mathrm{MoS}_{2}$ bulk reference are also displayed. $\mathbf{h}$ The atomic model of the (Co, $\mathrm{Cr}$ )-MoS 2 monolayer

enhancement in saturation magnetization and a 35 -fold increase in PL intensity relative to the pristine monolayer $\mathrm{MoS}_{2}$. A single flake of such a monolayer was utilized to construct a back-gate field effect transistors (FET) device. A positive magnetic resistance (MR) of $35 \%$ is observed at room-temperature. We expect that this work may extend the applications of two-dimensional chalcogenides into spintronics, valleytronic, and photoelectric devices.

\section{Results}

Sample preparation and characterization. The (Co, Cr) $-\mathrm{MoS}_{2}$ monolayer was grown on $300-\mathrm{nm}$ thick $\mathrm{SiO}_{2} / \mathrm{Si}$ substrates prepared by chemical vapor deposition (CVD), which employed (Co, Cr)-co-doped $\mathrm{MoO}_{3}$ powders and pure sulphur as reactant materials (see the Methods section and Supplementary Fig. 1 for details). Compared with the case using the separate transitionmetal oxides and $\mathrm{MoO}_{3}$ as the precursors, this method is beneficial to the homogeneous mixing of $\mathrm{Co}, \mathrm{Cr}$, and $\mathrm{MoO}_{3}$ at the molecular level (Supplementary Figs. 2-5) ${ }^{28}$. The homogeneously mixed $\mathrm{Co}$ and $\mathrm{Cr}$ atoms entering the lattice of $\mathrm{MoO}_{3}$ possibly adopt similar coordination structure to that of Mo atoms. Thus, the formation of $(\mathrm{Co}, \mathrm{Cr})-\mathrm{MoS}_{2}$ nuclei avoids the precipitation of metallic $\mathrm{Co}$ and $\mathrm{Cr}$ from the preformed $\mathrm{MoS}_{2}$ monolayers driven by the self-purification effect. Depending on their different reactivity, $\mathrm{Co}$ and $\mathrm{Cr}$ can occupy their favorable spatial positions in the monolayer $\mathrm{MoS}_{2}$. Pristine monolayer $\mathrm{MoS}_{2}, \mathrm{Co}-\mathrm{MoS}_{2}$, and $\mathrm{Cr}-\mathrm{MoS}_{2}$ monolayers were also prepared using the CVD method.

The morphology and crystal structure of these as-obtained monolayers are shown by the optical microscope, atomic force microscope (AFM), transmission electron microscopy (TEM) images in Fig. 2. In the optical microscope (Fig. 2a), the domains with an average size of $\sim 20 \mu \mathrm{m}$ are clearly seen with a homogeneous color together with flakes having an equilateral triangular shape. The AFM image in Fig. $2 \mathrm{~b}$ further indicates the $\sim 0.78 \mathrm{~nm}$ thickness of the as-grown flake, revealing the monolayer morphology of the as-obtained (Co, Cr)- $\mathrm{MoS}_{2}$ as reported in the previous studies ${ }^{29,30}$. As shown by the high-resolution transmission electron microscopy (HRTEM) image (Fig. 2c), the $(\mathrm{Co}, \mathrm{Cr})-\mathrm{MoS}_{2}$ monolayer is of the hexagonal lattice structure with the lattice spacing of 0.27 and $0.16 \mathrm{~nm}$ assigned to the (100) and (110) planes of the $2 \mathrm{H}-\mathrm{MoS}_{2}$ phase. This is further supported by the corresponding selected area electron diffraction (SAED) pattern (inset of Fig. 2c). Meanwhile, the energy-dispersive X-ray (EDX) mapping images in Fig. 2d qualitatively reveal that the chemical composition of the samples include not only the Mo and $\mathrm{S}$ elements, but also the $\mathrm{Cr}$ and Co elements. The EDX spectroscopy also indicates that besides $\mathrm{Co}$ and $\mathrm{Cr}$, there is no other metal impurity that could induce FM (Supplementary Fig. 6). Moreover, the $\mathrm{Mo} / \mathrm{Co} / \mathrm{Cr}$ molar ratio, as determined by the inductively coupled plasma atomic emission spectrometry (ICP-AES) analysis, is 1: 0.01: 0.003 (Supplementary Fig. 7 and Supplementary Table 1). To identify the location of $\mathrm{Cr}$ and $\mathrm{Co}$ atoms within the monolayer $\mathrm{MoS}_{2}$, high-angle annular dark-field scanning transmission electron microscopy (HAADF-STEM) measurements were performed. From Fig. 2e, we can see randomly distributed darker spots marked by red circles and brighter spots marked by yellow circles in the medium white (Mo atoms) atomic lattice. The corresponding cross-sectional intensity of the atom contrast in Fig. $2 \mathrm{f}$ reveals that the $\mathrm{Cr}$ or Co atoms possibly anchor on Mo atop sites or substitute for Mo atoms.

Next, X-ray absorption fine structure (XAFS) measurements were carried out to detect the real occupation positions of the Co and $\mathrm{Cr}$ atoms. XAFS has been a widely used technique for determination of the spatial occupations of doping elements due to its sensitivity to local atomic and electronic structures ${ }^{31-33}$. Figure $2 \mathrm{~g}$ displays the Fourier transformed (FT) curve of the $\mathrm{Cr}$ and Co $K$-edge extended X-ray absorption fine structure (EXAFS) $k \chi(k)$ functions for $(\mathrm{Co}, \mathrm{Cr})-\mathrm{MoS}_{2}$ monolayer. As reference, the Mo K-edge function of bulk $\mathrm{MoS}_{2}$ reference is also plotted. Evidently, the Co and Cr K-edge FT curves of the (Co, Cr)- $\mathrm{MoS}_{2}$ monolayer display considerably different features. Like the Mo $K$ edge FT curve of bulk $\mathrm{MoS}_{2}$, the FT at Co $K$-edge exhibits two prominent coordination peaks at $1.9 \AA$ (Co-S coordinations) and $2.8 \AA$ (Co-Mo coordinations), suggesting the substitutional doping of Co in the $\mathrm{MoS}_{2}$ host. However, only the $1.9 \AA$ peak is prominent in the FT curve at $\mathrm{Cr} K$-edge. In combination with the HAADF result, it can be inferred that the $\mathrm{Cr}$ atoms are anchored on the Mo atop sites of the monolayer $\mathrm{MoS}_{2}$. Based on the structural model of Co substituting for the interior Mo sites and $\mathrm{Cr}$ anchored on the atop Mo sites, we calculated the X-ray 
a

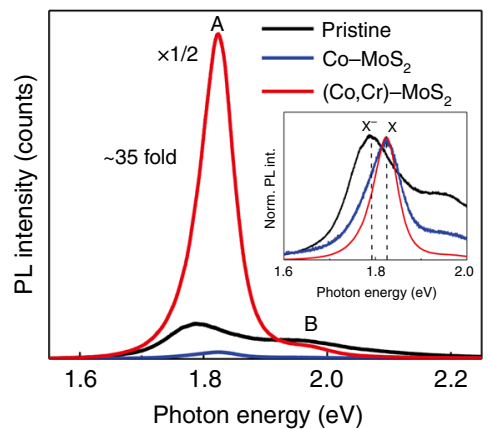

c

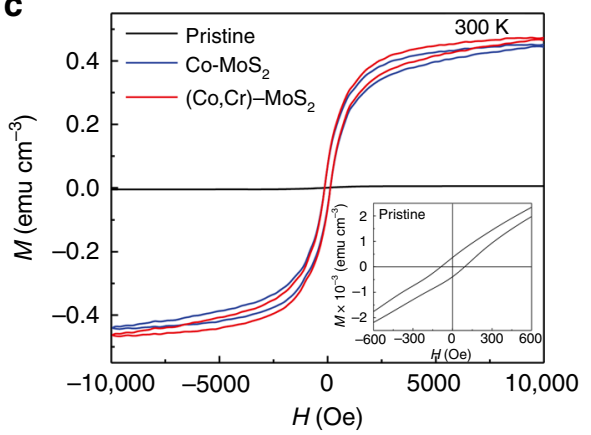

b

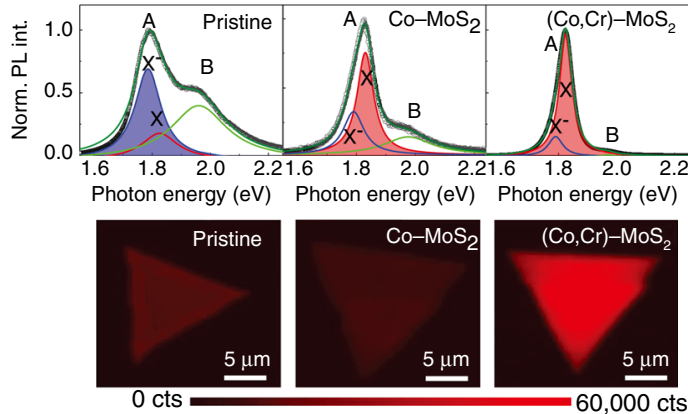

d

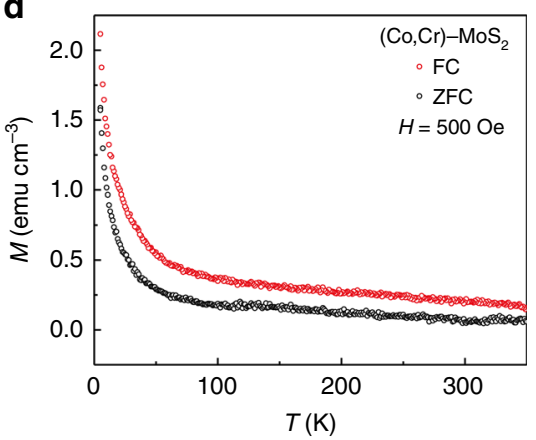

Fig. 3 Characterization of luminescent and magnetic properties. a Unnormalized PL spectra of pristine monolayer $\mathrm{MoS}_{2}, \mathrm{Co}_{-}-\mathrm{MoS}{ }_{2}$, and $(\mathrm{Co}, \mathrm{Cr})-\mathrm{MoS}{ }_{2}$. The inset shows the normalized PL spectra. b Analysis of the PL spectral shapes and PL intensity mappings, and c Magnetization vs magnetic field ( $M-H)$ curves. d Temperature-dependence of magnetization $(M-T)$ curves of $(\mathrm{Co}, \mathrm{Cr})-\mathrm{MoS}_{2}$ with FC and ZFC process

absorption near-edge structure (XANES) spectra at both Co and $\mathrm{Cr} K$-edges by using the ab initio code FEFF8 (Supplementary Fig. 8$)^{34}$. The calculations could well reproduce the spectral features of the experimental data, affording further support to the validity of the structure illustrated in Fig. $2 \mathrm{~h}$. These results lead us to conclude that monolayer $\mathrm{MoS}_{2}$ with different occupation sites of $\mathrm{Co}$ and $\mathrm{Cr}$ atoms is successfully obtained by CVD: the $\mathrm{Cr}$ atoms are anchored on the Mo atop sites of the monolayer $\mathrm{MoS}_{2}$, while Co atoms substitute for Mo in the monolayer $\mathrm{MoS}_{2}$ host, as indicated by the atomic model schematically shown in Fig. $2 \mathrm{~h}$.

Coexistence of luminescent and magnetic properties. To investigate the influence of $\mathrm{Co}, \mathrm{Cr}$ atoms on the luminescent and magnetic properties of monolayer $\mathrm{MoS}_{2}$, PL spectra and magnetization $(M-H)$ curves were measured at room temperature. Representative PL spectra and the integrated intensity maps of the normalized PL spectra for pristine $\mathrm{MoS}_{2}, \mathrm{Co}-\mathrm{MoS}_{2}$ and (Co, $\mathrm{Cr})-\mathrm{MoS}_{2}$ monolayers are displayed in Fig. 3a, b, respectively. We first show the unnormalized PL spectra which allow us to compare directly their PL intensities. Obviously, the pristine monolayer $\mathrm{MoS}_{2}$ shows weak PL intensity (Fig. 3a), possibly arising from sulfur vacancies induced electrons that transform most of the excitons $(\mathrm{X})$ into negative trions $\left(\mathrm{X}^{-}\right)(\text {Fig. } 3 \mathrm{~b})^{17}$. Interestingly, the presence of sulfur vacancy, which decreases the PL intensity, induces ferromagnetic ordering (saturation magnetization of about $0.004 \mathrm{emu} \mathrm{cm}^{-3}$ ) in the non-magnetic pristine monolayer $\mathrm{MoS}_{2}$ (Fig. 3c) 20,35,36. After Co incorporation, the saturation magnetization is greatly enhanced to $\sim 0.4 \mathrm{emu} \mathrm{cm}^{-3}$ $\left(0.3 \mu_{\mathrm{B}}\right.$ per Co atom), 90 times higher than that of the pristine monolayer $\mathrm{MoS}_{2}$. And seen from Supplementary Fig. 9, the saturation magnetization of $\mathrm{Co}-\mathrm{MoS}_{2}$ monolayer is decreased from $\sim 1.0 \mathrm{emu} \mathrm{cm}^{-3}$ at $5 \mathrm{~K}$ to $\sim 0.4 \mathrm{emu} \mathrm{cm}^{-3}$ at room-temperature, a feature like those observed in a diversity of transitionmetal doped diluted magnetic oxides ${ }^{37}$. However, the PL intensity of the Co- $\mathrm{MoS}_{2}$ becomes weaker (Fig. 3a), in spite of the dominant exciton peak (X) (Fig. 3b). The main reason is that the substitutional Co atoms usually act as nonradiative recombination centers and significantly quench the luminescence. A similar change in the PL intensity was also observed by Zhang et al. ${ }^{19}$ on Mn-doped monolayer $\mathrm{MoS}_{2}$. Surprisingly, after incorporating $\mathrm{Cr}$ into the $\mathrm{Co}-\mathrm{MoS}_{2}$ monolayer, the PL intensity map shows a bright red color, and the PL intensity shows a 35 -fold enhancement in relation to that of the pristine $\mathrm{MoS}_{2}$. Moreover, the $\mathrm{Cr}$ incorporation does not cause a noticeable change in the saturation magnetization $\left(1.0 \mathrm{emu} \mathrm{cm}^{-3}(5 \mathrm{~K}), 0.4 \mathrm{emu} \mathrm{cm}^{-3}(300 \mathrm{~K})\right.$, Fig. 3c and Supplementary Fig. 9). Therefore, $\mathrm{Cr}$ atoms do not change the magnetic ordering of the $\mathrm{Co}-\mathrm{MoS}_{2}$ monolayer, because of the low $\mathrm{Cr}$ content and its small contribution to the total magnetic moment. This is in agreement with the firstprinciples density-functional calculations by Yun et al. ${ }^{38}$, who predicted that doping Cr into monolayer $\mathrm{MoS}_{2}$ could not activate strong magnetism. From the comparison of the magnetism and PL intensity changes (Supplementary Fig. 10), we can conclude that synergetic incorporation of two TM elements can indeed realize the coexistence of outstanding optical and robust FM in monolayer $\mathrm{MoS}_{2}$.

To enrich our understanding of the magnetic behaviors of the doped $\mathrm{MoS}_{2}$, we have measured the temperature-dependence of magnetization $(M-T)$ curves of all samples. Zero-field-cooled (ZFC) and field cooled (FC) measurements were performed on the representative samples (Fig. 3d). From the $M-T$ and $M-H$ data, besides the FM, diamagnetism and paramagnetism could be observed (see Supplementary Fig. 11 for details of the analysis of these magnetic components). The ZFC-FC curves display similar features to those of dilute ferromagnetic oxides and suggest their similar intrinsic magnetic behaviors ${ }^{37}$. More importantly, the ZFC-FC curves show the nonexistence of superparamagnetic phase transition and progressive freezing of spins in our doped $\mathrm{MoS}_{2}$ samples, thus excluding the precipitation of the Co clusters. 

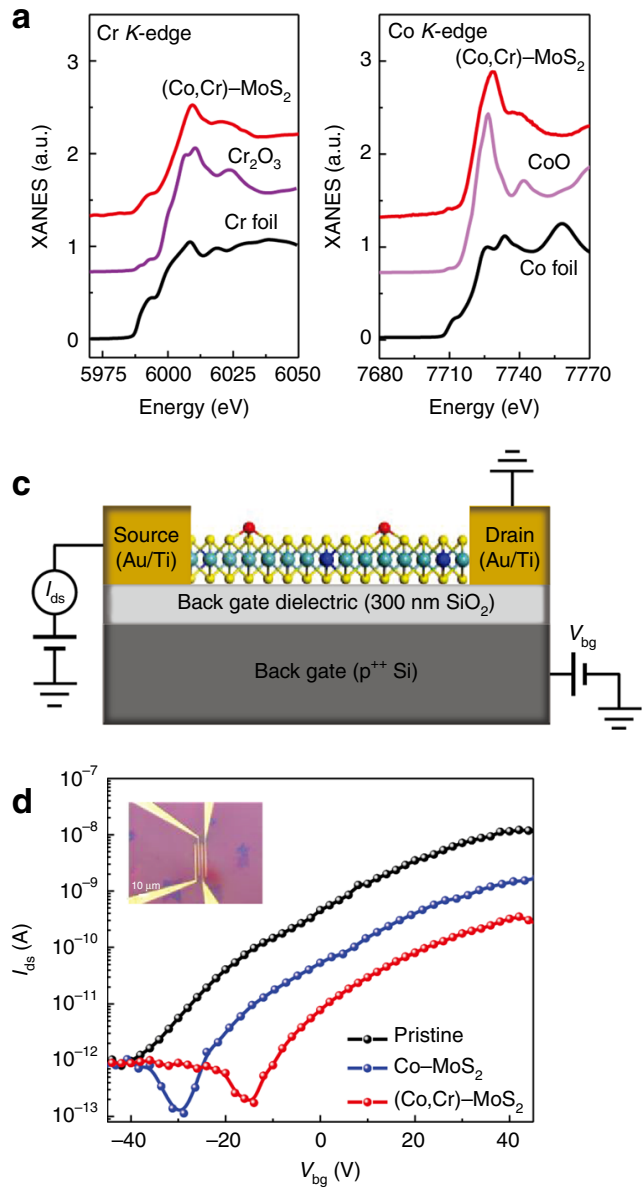

b
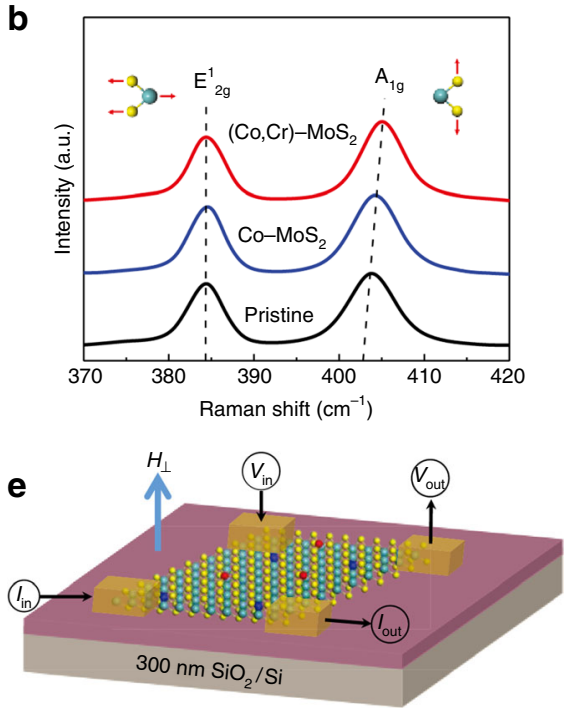

f

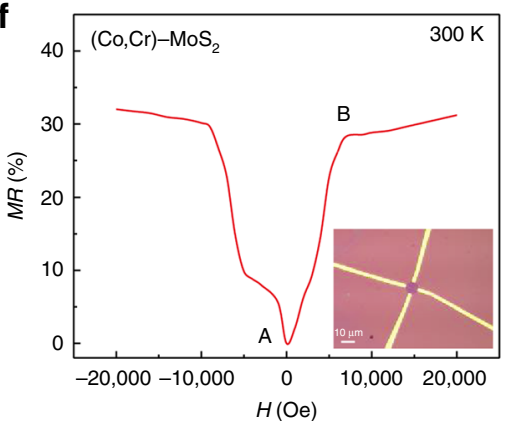

Fig. 4 The effect of incorporating alien TM atoms into $\mathrm{MoS}_{2}$ monolayer. a $\mathrm{Co}$ and $\mathrm{Cr}$ K-edge XANES spectra of (Co, $\mathrm{Cr}$ )-MoS 2 monolayer and $\mathrm{Cr}$ foil, $\mathrm{Cr}_{2} \mathrm{O}_{3}$, Co foil, $\mathrm{CoO}$. b Raman spectra of pristine monolayer $\mathrm{MoS}_{2}, \mathrm{Co}_{-}-\mathrm{MoS}_{2}$, and (Co, Cr)-MoS . c Schematic illustration of the back-gate FET device. d Drain-source current $\left(I_{\mathrm{ds}}\right)$ versus gate voltage $\left(V_{\mathrm{bg}}\right)$ transfer characteristics at $V_{\mathrm{ds}}=1 \mathrm{~V}$. e Schematic illustration of van der Pawl geometry in MR measurement. $\mathbf{f}$ Magnetic-field-dependent resistance of $(\mathrm{Co}, \mathrm{Cr})-\mathrm{MoS}_{2}$ at $300 \mathrm{~K}$

If we assume that the magnetism were originated from ferromagnetic metallic Co clusters, from the measured saturation magnetization $\left(0.3 \mu_{\mathrm{B}}\right.$ per atom $)$ and that $\left(1.7 \mu_{\mathrm{B}}\right.$ per atom $)$ of a Co ion in Co metal clusters, we could estimate that $15 \%$ of all the doped Co atoms are in the form of Co clusters. Such a high level of Co clusters could be easily detected by XANES ${ }^{39,40}$. However, seen from Fig. 4a, our XANES data do not show any characteristic peaks of metallic Co cluster. From the vibrating sample magnetometer (VSM) measurements, the Curie temperature $\left(T_{\mathrm{C}}\right)$ of $(\mathrm{Co}, \mathrm{Cr})-\mathrm{MoS}_{2}$ monolayer is around $500 \mathrm{~K}$ (Supplementary Fig. 12), which is much lower than the $T_{\mathrm{C}}$ of metallic Co clusters. Besides, in our EDX spectroscopy and ICP-AES measurements, detectable impurity elements that may trigger the magnetism are only Co and $\mathrm{Cr}$ (see Supplementary Fig. 6, 7).

Summarizing the above results, it can be concluded that the magnetic behavior of our doped $\mathrm{MoS}_{2}$ originates from the substitutional Co ions. Due to the low Co content and the existence of sulphur vacancy, the room-temperature FM could be understood within the framework of bound magnetic polaron mechanism ${ }^{41}$. That is, the spins of the localized defects (electrons bond by $\mathrm{S}$ vacancy) align those of the nearby Co ions, producing an effective magnetic field and activating the ferromagnetic interactions between $\mathrm{Co}$ ions within the polaron radius (Supplementary Fig. 13). It is worthy of note that the coercive field for a single $\mathrm{MoS}_{2}$ layer is close to that of a large number of films. This suggests that the FM observed in a large number of films really originates from single $\mathrm{MoS}_{2}$ monolayers, rather than from the interactions between different $\mathrm{MoS}_{2}$ monolayers (see Supplementary Fig. 14 for the magneto-optical Kerr effect (MOKE) signal). It reflects the fact that the FM in a large number of $\mathrm{MX}_{2}$ films actually comes from the FM of a single layer.

Effect of incorporating alien TM atoms. Next, XANES spectra were employed to understand why incorporation of $\mathrm{Co}$ and $\mathrm{Cr}$ could effectively tune the magnetic and optical properties of monolayer $\mathrm{MoS}_{2}$. As shown in Fig. 4a, the $\mathrm{Cr} \mathrm{K}$-edge XANES spectrum shows an absorption edge position quite close to $\mathrm{Cr}^{3+}$ in $\mathrm{Cr}_{2} \mathrm{O}_{3}$ but different from $\mathrm{Cr}^{0}$ in $\mathrm{Cr}$ foil, indicating the 3 + valence of $\mathrm{Cr}$ in the monolayer $\mathrm{MoS}_{2}$. On the other hand, the Co absorption edge position is close to that of $\mathrm{CoO}$ compound (Fig. 4a), implying the $\mathrm{Co}^{2+}$ valence of the substitutional Co. The Raman spectra in Fig. 4b further show that associated with the doping of $\mathrm{Co}^{2+}$ and $\mathrm{Cr}^{3+}$ ions, the $\mathrm{A}_{1 \mathrm{~g}}$ peak (out-of-plane vibration mode) is blue shifted gradually, while the frequency of in-plane vibration mode $\left(\mathrm{E}_{2 \mathrm{~g}}^{1}\right.$ peak) is almost unaffected. This suggests that the electron carrier density in monolayer $\mathrm{MoS}_{2}$ is reduced upon incorporation of $\mathrm{Co}$ and $\mathrm{Cr}^{42,43}$. For getting further knowledge on this point, we characterized the electrical transport in monolayer $\mathrm{MoS}_{2}$ before and after Co incorporation and (Co, $\mathrm{Cr}$ )-incorporation. The schematic illustration and optical image of the back-gate FET device by using a single flake of the (Co, $\mathrm{Cr})-\mathrm{MoS}_{2}$ monolayer with the Ti/Au $(10 \mathrm{~nm} / 100 \mathrm{~nm})$ source and drain electrodes are shown in Fig. $4 \mathrm{c}$ and inset in Fig. 4d, 
respectively. Obviously, the threshold voltages $\left(V_{\mathrm{bg},}\right.$ th $)$ of $\mathrm{Co}-\mathrm{MoS}_{2}(-30 \mathrm{~V})$ and $(\mathrm{Co}, \mathrm{Cr})-\mathrm{MoS}_{2}(-15 \mathrm{~V})$ substantially shift compared to that of pristine monolayer $\mathrm{MoS}_{2}(-40 \mathrm{~V})$ with lower current level, as shown in the source-drain current $\left(I_{\mathrm{ds}}\right)$ versus gate voltage $\left(V_{\mathrm{bg}}\right)$ transfer curves (Fig. $4 \mathrm{~d}$ and Supplementary Fig. 15). According to the parallel-plate capacitor model, the charge concentration can be calculated from the equation $n=$ $C_{\mathrm{ox}} \Delta V_{\mathrm{bg}} / e$, where $C_{\mathrm{ox}}=\varepsilon_{0} \varepsilon_{\mathrm{r}} / d_{\mathrm{ox}}, e=1.602 \times 10^{-19} \mathrm{C}$ is the elementary charge and $\Delta V_{\mathrm{bg}}=V_{\mathrm{bg}}-V_{\mathrm{bg}, \mathrm{th}^{4}}{ }^{44}$. At $V_{\mathrm{bg}}=0 \mathrm{~V}$, the charge concentration of the pristine $\mathrm{MoS}_{2}, \mathrm{Co}-\mathrm{MoS}_{2}$, and (Co, $\mathrm{Cr})-\mathrm{MoS}_{2}$ is $\sim 2.9 \times 10^{12}, 2.1 \times 10^{12}$, and $1.1 \times 10^{12} \mathrm{~cm}^{-2}$, respectively. Therefore, the Co and $\mathrm{Cr}$ in monolayer $\mathrm{MoS}_{2}$ act as the intrinsic $p$-type dopants and reduce the electron density. A consequence of the reduced electron density is the transition of the main part of the negatively charged trion $\left(\mathrm{X}^{-}\right)$into neutral exciton (X), as illustrated in Fig. 3b. Therefore, the relative increase in exciton content is the main reason for the enhanced $\mathrm{PL}$ intensity in the (Co, Cr) $-\mathrm{MoS}_{2}$ monolayer.

Based on the van der Pawl geometry, as schematically shown in Fig. 4e, under low magnetic field, the (Co, Cr) $-\mathrm{MoS}_{2}$ monolayer exhibits a positive MR with a surprising magnitude of $35 \%$ (Fig. 4f) at room-temperature. Besides, in the MR curve the positions of the two characteristic points $A$ and $B$, which reflect the coercive field and saturation field, match well with the coercive field $(\sim 100 \mathrm{Oe})$ and saturation field $(\sim 10 \mathrm{kOe})$ determined in the out-of-plane $M-H$ loop, respectively (Supplementary Fig. 16). It is worthy of note that the magnetic field needed for saturating the in-plane MR is lower than that for the out-ofplane MR (Supplementary Fig. 17). This is in line with the $M-H$ results shown in Supplementary Fig. 16, where the saturation fields decreases as the external magnetic field is changed from the out-of-plane to the in-plane direction. These results show the magnetic anisotropy of $(\mathrm{Co}, \mathrm{Cr})-\mathrm{MoS}_{2}$ with the easy magnetization axis parallel to the surface of monolayer. To the best of our knowledge, positive MR on $\mathrm{MoS}_{2}$ monolayer-based materials has been never observed before. Positive MR was previously observed on Co-doped $\mathrm{ZnO}$ nanowires with room-temperature FM by Yang et al. ${ }^{45}$, who interpreted it as a result of the electron redistribution between two FM-induced sub-bands in the presence of a magnetic field. In a single (Co, Cr)- $\mathrm{MoS}_{2}$ monolayer, anomalous Hall effect is observed (Supplementary Fig. 18). This measurement affords further evidence to the ferromagnetic behavior of the ( $\mathrm{Co}, \mathrm{Cr})-\mathrm{MoS}_{2}$ monolayer, and also to the magnetic interactions between the doped ions. We hypothesize that the $\mathrm{MR}$ is due to the broken time-reversal symmetry of the $\mathrm{MoS}_{2}$ monolayer by the magnetic exchange interaction between magnetic atoms, thus the two-fold valley degeneracy is lifted ${ }^{13,46}$. That is, the FM acts as a factor to manipulate the valley DOF as we expected. In ( $\mathrm{Co}, \mathrm{Cr})-\mathrm{MoS}_{2}$ monolayer, there emerges a large separation in the momentum space between two valleys with lifted degeneracy. At roomtemperature, the energy difference between these valleys is still higher than the Fermi energy, and the difference of the electron populations in these two valleys are still remarkable. Therefore, the positive MR remains at room-temperature. In other words, the positive $\mathrm{MR}$ for the (Co, Cr) $-\mathrm{MoS}_{2}$ monolayer is intimately related to the valley polarization induced by FM property.

\section{Discussion}

For an in-depth understanding of the origin of the coexisting robust magnetism and PL from the aspect of electronic band structure, we employed ABINIT software package to calculate the electronic structure of pristine $\mathrm{MoS}_{2}, \mathrm{Co}-\mathrm{MoS}_{2}$, and (Co, Cr)- $\mathrm{MoS}_{2}$ monolayers (Fig. 5a). This software package implements densityfunctional theory using a plane-wave basis set and generalized gradient approximation (GGA) with the Perdew-Burke-Enzerhoff (PBE) functional. The details of the calculations are included in the Methods section. The obtained density of states (DOS) are plotted in Fig. 5b. The calculated bandgap of pristine monolayer $\mathrm{MoS}_{2}$ is $1.65 \mathrm{eV}$, underestimated by merely $0.15 \mathrm{eV}$ compared with the experimental and calculated values previously 47,48 . The small difference indicates the validity of GGA for $\mathrm{MoS}_{2}$.

For $\mathrm{Co}-\mathrm{MoS}_{2}$ monolayer, a striking bandgap impurity band can be observed, and the spin polarization contributes to magnetism with a magnetic moment of $3 \mu_{\mathrm{B}}$ per cell, in agreement with the result by Wang et $\mathrm{al}^{49}$. The forementioned discussions indicate that the room-temperature ferromagnetic ordering of the sample originates from the substitutional Co atoms with non-zero

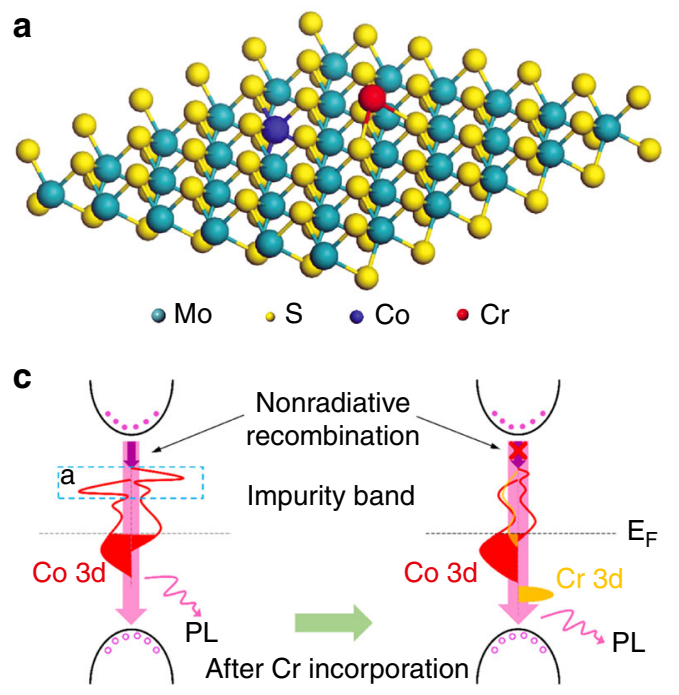

b

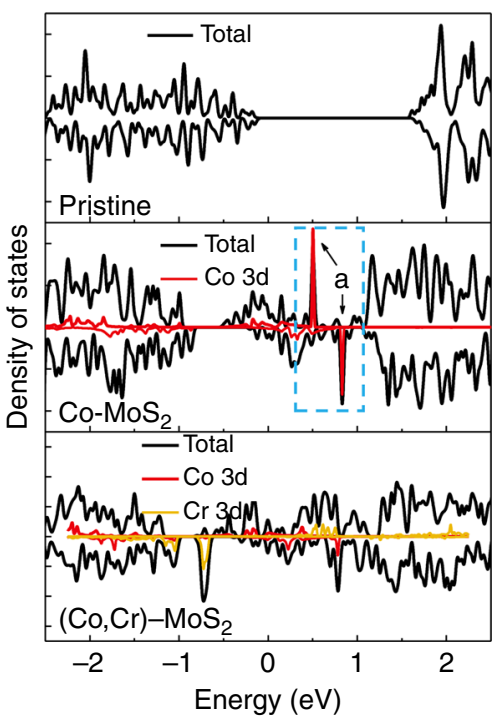

Fig. 5 The origin of the coexisting robust magnetism and PL. a Structure model for the DFT calculations on the ( $\mathrm{Co}, \mathrm{Cr})-\mathrm{MoS} 2$ monolayer. $\mathbf{b}$ Calculated density of states (DOS) of monolayer $\mathrm{MoS}_{2}$ : pristine $\mathrm{MoS}_{2}, \mathrm{Co}_{\mathrm{O}}-\mathrm{MoS}_{2}$, and (Co, Cr)-MoS monolayers. c Schematic band structure of monolayer (Co, $\mathrm{Cr}$ )$\mathrm{MoS}_{2}$ with impurity bands 
magnetic moments. However, the substitutional Co atoms also induce a localized impurity band $a$ within the bandgap (Fig. 5b). Due to the high state density, this impurity band provides a nonradiative de-exciting channel for the excited $\mathrm{CB}$ photoelectrons ${ }^{23}$. That is to say, the bandgap impurity band $a$ acts as the nonradiative recombination center and reduces the PL intensity (Fig. 5c). In contrast, the impurity band induced by $\mathrm{Cr}$ atoms does not lead to remarkable nonradiative recombination as revealed by the PL experiment, since the PL intensity of monolayer $\mathrm{MoS}_{2}$ is not reduced significantly by $\mathrm{Cr}$ incorporation (Supplementary Fig. 19). In fact, similar phenomenon has also been observed in $\mathrm{Cr}$-doped $\mathrm{ZnO}$ semiconductor ${ }^{50,51,52}$. These results indicate that $\mathrm{Cr}$ atoms, different from magnetic $\mathrm{TM}$ ions (such as $\mathrm{Mn}$ and $\mathrm{Co}$ ), do not reduce the luminescence of semiconductor hosts, possibly because of the lack of high-density bandgap impurity band. Incorporating $\mathrm{Cr}$ with Co causes a remarkable redistribution of Co-induced bandgap impurity; especially, the state density of localized impurity band $a$ is reduced significantly. Therefore, the $\mathrm{Cr}$ adatoms could suppress the nonradiative recombination caused by Co mono-doping (Fig. 5b, c), through electronic interactions between the $\mathrm{Cr}$ and Co in the $\mathrm{MoS}_{2}$ monolayer. Similar changes is also observed in the DOSs generated by using the local-density approximation (LDA) plus spin-orbit coupling (SOC) (Supplementary Fig. 20), in agreement with the GGA calculations. The Bader charge analysis further shows that on the average about $0.30 e$ electron is transferred from a $\mathrm{Cr}$ atom to the $\mathrm{Co}-\mathrm{MoS}_{2}$ monolayer. On the other side, the solo-doping of $\mathrm{Cr}$ could not improve remarkably the PL intensity of the $\mathrm{MoS}_{2}$ monolayer, as inferred from Supplementary Fig. 20. Thus the synergetic incorporation of Co and $\mathrm{Cr}$ breaks the physical mechanism that excludes the coexistence of magnetic ordering and luminescent feature in $\mathrm{MoS}_{2}$ monolayer.

In conclusion, using the monolayer $\mathrm{MoS}_{2}$ as an example, we have experimentally demonstrated that the synergetic interaction of two alien TM elements provides an effective way to realize the coexistent optical and ferromagnetic properties in twodimensional chalcogenide semiconductors. As shown by a detailed study of structural, optical and magnetic properties, this idea is successfully applied to $\mathrm{MoS}_{2}$ monolayer incorporated by $\mathrm{Co}$ and $\mathrm{Cr}$ atoms. As compared with the pristine monolayer $\mathrm{MoS}_{2}$, the saturation magnetization and PL intensity of the (Co, $\mathrm{Cr}$ )- $\mathrm{MoS}_{2}$ monolayer is enhanced by 90 and 35 times, respectively. A single flake of the (Co, Cr)- $\mathrm{MoS}_{2}$ monolayer was used to construct a back-gate FET device; the electricity measurement shows the intrinsic p-type doping of $\mathrm{Co}$ and $\mathrm{Cr}$ in $\mathrm{MoS}_{2}$. Based on the van der Pawl geometry, a positive MR (35\%) is observed at room-temperature, which arises from the p-type doping and is intimately related to the valley polarization induced by the FM. We expect that this idea can be generalized to tune the optical and magnetic properties of other two-dimensional semiconducting materials, and it opens up possibilities for simultaneous polarization and detection of the valley DOF for future valleytronics applications.

\footnotetext{
Methods

Precursors prepared. To synthesize monolayer (Co, Cr)- $\mathrm{MoS}_{2}$, the precursors of $\mathrm{Co}-\mathrm{MoO}_{3}$ and $\mathrm{Cr}-\mathrm{MoO}_{3}$ were prepared by the sol-gel method. We dissolved 0.856 $\mathrm{g}$ of hexaammonium heptamolybdate tetrahydrate $\left(\mathrm{NH}_{4}\right)_{6} \mathrm{Mo}_{7} \mathrm{O}_{24} \cdot 4 \mathrm{H}_{2} \mathrm{O}, 45 \mathrm{mg}$ of cobaltous nitrate hexahydrate $\mathrm{Co}(\mathrm{NO})_{3} \cdot 6 \mathrm{H}_{2} \mathrm{O}$ and $10 \mathrm{mg}$ of $\mathrm{Cr}(\mathrm{NO})_{3} \cdot 9 \mathrm{H}_{2} \mathrm{O}, 2.1 \mathrm{~g}$ of citric acid in deionized water. The mixed solution was stirred to form sol and dried at $110 \mathrm{C}$ for $2 \mathrm{~h}$. Then the obtained gel was heated at $150{ }^{\circ} \mathrm{C}$ for $12 \mathrm{~h}$, calcined at $550{ }^{\circ} \mathrm{C}$ for $5 \mathrm{~h}$, and then cooled to room temperature naturally. The $\mathrm{Cr}-\mathrm{MoO}_{3}$ was prepared in the same way, namely, a solution of $0.856 \mathrm{~g}$ of hexaammonium heptamolybdate tetrahydrate $\left(\mathrm{NH}_{4}\right)_{6} \mathrm{Mo}_{7} \mathrm{O}_{24} \cdot 4 \mathrm{H}_{2} \mathrm{O}, 10 \mathrm{mg}$ of chromic nitrate nonahydrate $\mathrm{Cr}(\mathrm{NO})_{3} \cdot 9 \mathrm{H}_{2} \mathrm{O}, 2.1 \mathrm{~g}$ of citric acid in deionized water was stirred to form sol and dried at $110^{\circ} \mathrm{C}$ for $2 \mathrm{~h}$. Then the obtained gel was heated at $90^{\circ} \mathrm{C}$ for
} $12 \mathrm{~h}$ and calcined at $600^{\circ} \mathrm{C}$ for $5 \mathrm{~h}$.
CVD of monolayer $\mathbf{M o S}_{\mathbf{2}} \cdot \mathrm{MoS}_{2}$ monolayers were synthesized by CVD method on $300 \mathrm{~nm} \mathrm{SiO} / / \mathrm{Si}$ substrates. The substrates were cleaned in acetone and isopropanol, then soaked in $\mathrm{H}_{2} \mathrm{SO}_{4} / \mathrm{H}_{2} \mathrm{O}_{2}$ (3:1) for $1 \mathrm{~h}$ and treated by $\mathrm{O}_{2}$ plasma for $10 \mathrm{~min}$. As shown in Supplementary Fig. 1 , the as-obtained $\mathrm{MoO}_{3}$ precursors and sulfur were loaded in two crucibles in 2-inch two-zone tube furnace, with sulfur located upstream and the substrates placed face-down above the crucible containing $\mathrm{MoO}_{3}$ precursors. The tube was pumped and flushed with $\mathrm{Ar}$ carrier gas three times and purged for 30 min with $200 \mathrm{sccm}$ Ar. Then the $\mathrm{MoO}_{3}$ precursor zone was heated to $850^{\circ} \mathrm{C}$ in $70 \mathrm{~min}$ with $100 \mathrm{sccm} \mathrm{Ar}$ at atmosphere pressure. When the temperature reached $650^{\circ} \mathrm{C}$, sulfur precursor zone was heated to $180^{\circ} \mathrm{C}$ and the sulfur gas was introduced. After the growth was finished for $5 \mathrm{~min}$, the furnaces were cooled down naturally.

Transfer of CVD grown MoS $_{\mathbf{2}}$. The polymethyl methacrylate (PMMA) liquid was spin-coated on $\mathrm{SiO}_{2} / \mathrm{Si}$ substrate grown with $\mathrm{MoS}_{2}$ monolayers at $4000 \mathrm{rpm}$ for 30 $\mathrm{s}$, and baked at $180^{\circ} \mathrm{C}$ for $2 \mathrm{~min}$. Then, $1 \mathrm{M} \mathrm{KOH}$ was used to etch the $\mathrm{SiO}_{2}$ layer and the obtained PMMA-MoS ${ }_{2}$ film was rinsed in deionized water three times. After that, the film was transferred and dried on the target substrates. The residue PMMA was washed with acetone.

Device fabrication and measurement. The back-gated FETs devices were fabricated on $300 \mathrm{~nm}$ thick $\mathrm{SiO}_{2} / \mathrm{Si}$ chips to estimate the carrier density. The transferred $\mathrm{MoS}_{2}$ samples were spin-coated with PMMA at $2500 \mathrm{rpm}$ for $1 \mathrm{~min}$, and baked at 180 ansferred and dried on the target substrates. The rehen patterned by electronbeam lithography with a bilayer PMMA stack and developed in methyl isobutyl ketone (MIBK)/isopropanol (IPA) (MIBK: IPA = 1:3) solution for 3 min. A subsequent electron-beam deposition of Ti/Au $(10 \mathrm{~nm} / 100 \mathrm{~nm})$ was followed and lifted-off in acetone. The devices were annealed in a vacuum tube furnace at 2500/ isopropanosccm Ar flow and $10 \mathrm{sccm} \mathrm{H}_{2}$ flow for two hours. The obtained FETs were measured at room temperature under atmosphere pressure with a Lakeshore probe station equipped with Keithley 4200-SCS semiconductor characterization system. The van der Pawl geometry and Hall bar were fabricated by a two-step lithography. The patterns were obtained by $\mathrm{O}_{2}$ plasma etching after the first step lithography. The MR and Anomalous Hall Effect were measured in a Physical Property Measurement System (PPMS) system (Quantum Design).

Material characterization. The TEM measurement was carried out on a JEM$2100 \mathrm{~F}$ field emission electron microscope at an acceleration voltage of $200 \mathrm{kV}$. The high-resolution TEM (HRTEM) and corresponding EDX mapping analyses were performed on a JEOL JEMARF200F TEM/STEM with a spherical aberration corrector. High-resolution high-angle annular dark-field (HAADF) scanning transmission electron microscopy (STEM) was performed in a JEOL ARM200F with STEM aberration (Cs) corrector operated at $80 \mathrm{kV}$. The samples were transferred in nitrohydrochloric acid and diluted. Mo, Co, and $\mathrm{Cr}$ concentrations were determined by inductively coupled plasma atomic emission spectrometry (ICP-AES, Jarrel Ash model 955). The AFM image was taken with Veeco DI Nanoscope MultiMode V scanning probe microscope in tapping mode. The $\mathrm{Cr} K$ edge and Co $K$-edge XAFS spectra were measured at the 1W1B beamline of the Beijing Synchrotron Radiation Facility (BSRF), China. Due to the small amount of Co and Cr dopants, XAFS data was acquired in a fluorescence geometry using 32element germanium detector (Canberra). And the Mo K-edge XAFS spectra were collected in transmission mode at the BL14W1 beamline of the Shanghai Synchrotron Radiation Facility (SSRF), China. Magnetization studies were carried out using a superconducting quantum interference device (SQUID) magnetometer. Magnetic-field- and temperature-dependent magnetizations were measured by SQUID over a temperature range of $5-400 \mathrm{~K}$ at 500 Oe and fields up to $4 \mathrm{~T}$, which were adjusted to be perpendicular and parallel to the surface of the sample, separately. Magnetization per formula unit for the monolayer was determined from the magnetization divided by the number of formula units calculated from the substrate size times the percentage of surface covered by a single layer. The diamagnetic and paramagnetic contributions were subtracted from the raw data to yield the ferromagnetic hysteresis loop. The in-plane MOKE hysteresis loops was measured by NanoMOKE2 from Quantum Design with a $50 \mathrm{~mW}(660 \mathrm{~nm})$ laser source with a spot size $\sim 5 \mu \mathrm{m}$.

DFT calculation details. The DFT calculations were performed with the spinpolarized density function theory implemented in ABINIT soft package ${ }^{52,53}$. Electron-ion interaction was processed with the projected augmented wave (PAW) method. The GGA with the PBE functional was used to describe electron exchange-correlation. Van der Waals interaction correction was performed with DFT-D2 method ${ }^{54}$. A kinetic energy cutoff of $800 \mathrm{eV}$ and a $5 \times 5 \times 1 k$-grid for Brillouin zone sampling were adopted after detailed convergence tests. A $6 \times 6 \times 1$ hexagonal supercell of $\mathrm{MoS}_{2}$ monolayer was employed to model the Co substitution and $\mathrm{Cr}$ adsorption according to the supercell size convergence test results. A vacuum layer of $18 \AA$ was placed between adjacent $\mathrm{MoS}_{2}$ monolayers to prevent interlayer interaction. The Mo atop site which was energetically favorable adsorption site for TM ion adsorption on $\mathrm{MoS}_{2}$ substrates was considered. The adsorption structures were optimized with BGFS method until the maximum force on each atom was less than $0.1 \mathrm{meV} \AA^{-1}$. For comparison, the DOSs were also 
calculated using LDA plus SOC, as shown in Supplementary Fig. 20. The same reduction on state density of localized impurity band $a$ upon $\mathrm{Cr}$ co-doping is captured, which confirms our claim that the Cr doping causes significant redistribution of Co-induced bandgap impurity. The obtained DOS is not spin polarized because when SOC was on, the up and down spin channels cannot be distinguished.

It is worthy of note that in order to examine the convergence of our GGA calculations, the DOSs were calculated for $3 \times 3 \times 1,4 \times 4 \times 1,5 \times 5 \times 1$, and $6 \times 6 \times$ 1 monolayer $\mathrm{MoS}_{2}$ supercell with one Mo atom substituted by one Co atom and one $\mathrm{Cr}$ adatom attached. The corresponding $\mathrm{Mo}$ : $\mathrm{Co}: \mathrm{Cr}$ elemental ratios are 100:11:11, 100: 6.3: 6.3, 100: 4.0: 4.0, and 100: 2.8: 2.8 for these supercells, respectively. No significant changes on the impurity bands in the bandgap is observed when the supercell was expanded from $4 \times 4 \times 1$ to $5 \times 5 \times 1$, and $6 \times 6 \times$ 1 , which indicates that $4 \times 4 \times 1$ supercell is large enough to eliminate the selfinteraction between dopant atoms and to present the electronic modification of dilute dopants, in agreement with the previous result by Cheng et. $\mathrm{al}^{55}$. Therefore, the elemental concentration used in our DOS calculations for a $6 \times 6 \times 1$ monolayer $\mathrm{MoS}_{2}$ supercell could well reflect the dilute dopant concentrations ( $1 \% \mathrm{Co}, 0.3 \%$ $\mathrm{Cr}$ ) in our samples.

Details of XANES calculations. The structure models for Co substituting for the interior Mo sites and $\mathrm{Cr}$ anchored on the atop Mo sites were built up to model structural characters (Co, Cr)-MoS 2 . The atomic structures of the model was relaxed by optimizing the total energy; when the total energy convergence of up to $10^{-6} \mathrm{eV}$ was achieved we calculated the XANES spectra for the finally obtained atomic structure. To achieve convergence of calculation, a model consisting of 251 atoms was used for model structure. The total scattering potentials including a fully relaxed core-hole were obtained iteratively, by successive calculations of the potential until self-consistency was reached. Based on this scattering potential, the final states of the excited photoelectron were then calculated. The Hedin-Lundqvist model of exchange potential with a $0.3 \mathrm{eV}$ shift and additional broadening of $0.2 \mathrm{eV}$ was used to give a closest match between the simulated and experimental spectra.

\section{Data availability}

The data that support the findings of this study are available from the corresponding authors upon reasonable request.

Received: 11 April 2018 Accepted: 18 March 2019

Published online: 05 April 2019

\section{References}

1. Lee, J. et al. Valley magnetoelectricity in single-layer $\mathrm{MoS}_{2}$. Nat. Mater. 16, 887-891 (2017).

2. Kim, J. et al. Ultrafast generation of pseudo-magnetic field for valley excitons in $\mathrm{WSe}_{2}$ monolayers. Science 346, 1205-1208 (2014).

3. Zhang, Z. W. et al. Robust epitaxial growth of two-dimensional heterostructures, multiheterostructures, and superlattices. Science 357, 788-792 (2017).

4. Wang, Y. et al. Structural phase transition in monolayer $\mathrm{MoTe}_{2}$ driven by electrostatic doping. Nature 550, 487-491 (2017).

5. Sahoo, P. K. et al. One-pot growth of two-dimensional lateral heterostructures via sequential edge-epitaxy. Nature 553, 63-67 (2018).

6. Bonilla, M. et al. Strong room-temperature ferromagnetism in $\mathrm{VSe}_{2}$ monolayers on van der Waals substrates. Nat. Nanotech 13, 289-293 (2018).

7. Zeng, $\mathrm{H}$. et al. Valley polarization in $\mathrm{MoS}_{2}$ monolayers by optical pumping. Nat. Nanotech 7, 490-493 (2012).

8. Cao, T. et al. Valley-selective circular dichroism of monolayer molybdenum disulphide. Nat. Commun. 3, 887 (2012).

9. Mak, K. F., McGill, K. L., Park, J. \& McEuen, P. L. The valley Hall effect in $\mathrm{MoS}_{2}$ transistors. Science 344, 1489-1492 (2014).

10. Saito, Y. et al. Superconductivity protected by spin-valley locking in ion-gated $\mathrm{MoS}_{2}$. Nat. Phys. 12, 144-149 (2016).

11. Srivastava, A. et al. Valley Zeeman effect in elementary optical excitations of monolayer $\mathrm{WSe}_{2}$. Nat. Phys. 11, 141-147 (2015).

12. Aivazian, G. et al. Magnetic control of valley pseudospin in monolayer $\mathrm{WSe}_{2}$. Nat. Phys. 11, 148-152 (2015).

13. Tong, W. Y., Gong, S. J., Wan, X. G. \& Duan, C. G. Concepts of ferrovalley material and anomalous valley Hall effect. Nat. Commun. 7, 13612 (2016).

14. Ye, Y. et al. Electrical generation and control of the valley carriers in a monolayer transition metal dichalcogenide. Nat. Nanotech 11, 597-602 (2016).

15. Xu, X. D., Yao, W., Xiao, D. \& Heinz, T. F. Spin and pseudospins in layered transition metal dichalcogenides. Nat. Phys. 10, 343-350 (2014).
16. Yang, H., Kim, S. W., Chhowalla, M. \& Lee, Y. H. Structural and quantumstate phase transition in van der Waals layered materials. Nat. Phys. 13, 931-937 (2017)

17. Mak, K. F. et al. Tightly bound trions in monolayer $\mathrm{MoS}_{2}$. Nat. Mater. 12, 207-211 (2013).

18. Mouri, S., Miyauchi, Y. \& Matsuda, K. Tunable photoluminescence of monolayer $\mathrm{MoS}_{2}$ via chemical doping. Nano Lett. 13, 5944-5948 (2013).

19. Zhang, K. et al. Manganese doping of monolayer $\mathrm{MoS}_{2}$ : the substrate is critical. Nano Lett. 15, 6586-6591 (2015).

20. Cai, L. et al. Vacancy-induced ferromagnetism of $\mathrm{MoS}_{2}$ nanosheets. J. Am. Chem. Soc. 137, 2622-2627 (2015).

21. Andriotis, A. N. \& Menon, M. Tunable magnetic properties of transition metal doped $\mathrm{MoS}_{2}$. Phys. Rev. B 90, 125304 (2014).

22. Amani, M. et al. Near-unity photoluminescence quantum yield in $\mathrm{MoS}_{2}$. Science 350, 1065-1068 (2015).

23. Wang, H., Zhang, C. \& Rana, F. Ultrafast dynamics of defect-assisted electronhole recombination in monolayer $\mathrm{MoS}_{2}$. Nano Lett. 15, 339-345 (2015).

24. Salmani, E. et al. The electronic, magnetic and optical properties of $\mathrm{ZnO}$ doped with doubles impurities (Cr, Fe): An LDA-SIC and Monte Carlo study. J. Magn. Magn. Mater. 422, 348-355 (2017).

25. Yan, W. S. et al. Mediating distribution of magnetic Co ions by Cr-codoping in (Co, Cr): ZnO thin films. Appl. Phys. Lett. 97, 042504 (2010).

26. Suh, J. et al. Reconfiguring crystal and electronic structures of $\mathrm{MoS}_{2}$ by substitutional doping. Nat. Commun. 9, 199 (2018).

27. Lin, Y. C. et al. Properties of individual dopant atoms in single-layer $\mathrm{MoS}_{2}$ : Atomic structure, migration, and enhanced reactivity. Adv. Mater. 26, 2857-2861 (2014)

28. Pereira, L. G. et al. Influence of doping on the preferential growth of $\mathrm{a}-\mathrm{MoO}_{3}$. J. Alloy. Compd. 459, 377-385 (2008).

29. Benameur, M. M. et al. Visibility of dichalcogenide nanolayers. Nanotech 22, 125706 (2011)

30. Zheng, J. et al. High-mobility multilayered $\mathrm{MoS}_{2}$ flakes with low contact resistance grown by chemical vapor deposition. Adv. Mater. 29, 1604540 (2017).

31. Suh, J. et al. Doping against the native propensity of $\mathrm{MoS}_{2}$ : degenerate hole doping by cation substitution. Nano Lett. 14, 6976-6982 (2014).

32. Sun, Z. et al. XAFS in dilute magnetic semiconductors. Dalton Trans. 42, 13779-13801 (2013)

33. Yan, W. et al. Realizing ferromagnetic coupling in diluted magnetic semiconductor quantum dots. J. Am. Chem. Soc. 136, 1150-1155 (2014).

34. Ankudinov, A. L., Ravel, B., Rehr, J. J. \& Conradson, S. D. Real-space multiplescattering calculation and interpretation of $\mathrm{x}$-ray-absorption near-edge structure. Phys. Rev. B 58, 7565-7576 (1998).

35. Shidpour, R. \& Manteghian, M. A density functional study of strong local magnetism creation on $\mathrm{MoS}_{2}$ nanoribbon by sulfur vacancy. Nanoscale 2 , 1429-1435 (2010).

36. Zheng, $\mathrm{H}$. et al. Tuning magnetism of monolayer $\mathrm{MoS}_{2}$ by doping vacancy and applying strain. Appl. Phys. Lett. 104, 132403 (2014).

37. Pan, F., Song, C., Liu, X., Yang, Y. \& Zeng, F. Ferromagnetism and possible application in spintronics of transition-metal-doped $\mathrm{ZnO}$ films. Mater. Sci. Eng. R. 62, 1-35 (2008).

38. Yun, W. S. \& Lee, J. D. Unexpected strong magnetism of $\mathrm{Cu}$ doped singlelayer $\mathrm{MoS}_{2}$ and its origin. Phys. Chem. Chem. Phys. 16, 8990-8996 (2014)

39. Griffin, K. A., Pakhomov, A., Wang, C. M., Heald, S. M. \& Krishnan, K. M. Intrinsic ferromagnetism in insulating cobalt doped anatase $\mathrm{TiO}_{2}$. Phys. Rev. Lett. 94, 157204 (2005)

40. Chambers, S. A. \& Farrow, R. F. New possibilities for ferromagnetic semiconductors. MRS Bull. 28, 729-733 (2003).

41. Coey, J., Venkatesan, M. \& Fitzgerald, C. Donor impurity band exchange in dilute ferromagnetic oxides. Nat. Mater. 4, 173-179 (2005).

42. Chakraborty, B. et al. Symmetry-dependent phonon renormalization in monolayer $\mathrm{MoS}_{2}$ transistor. Phys. Rev. B 85, 161403 (2012).

43. Sun, L. et al. Spin-orbit splitting in single-layer $\mathrm{MoS}_{2}$ revealed by triply resonant Raman scattering. Phys. Rev. Lett. 111, 126801 (2013).

44. Radisavljevic, B. \& Kis, A. Mobility engineering and a metal-insulator transition in monolayer $\mathrm{MoS}_{2}$. Nat. Mater. 12, 815-820 (2013).

45. Liang, W. J., Yuhas, B. D. \& Yang, P. D. Magnetotransport in Co-Doped ZnO nanowires. Nano Lett. 9, 892-896 (2009).

46. Singh, N. \& Schwingenschlogl, U. A route to permanent valley polarization in monolayer $\mathrm{MoS}_{2}$. Adv. Mater. 29, 1600970 (2017).

47. Cheiwchanchamnangij, T. \& Lambrecht, W. R. L. Quasiparticle band structure calculation of monolayer, bilayer, and bulk $\mathrm{MoS}_{2}$. Phys. Rev. B 85, 205302 (2012).

48. Mak, K. F. et al. Atomically thin $\mathrm{MoS}_{2}$ : a new direct-gap semiconductor. Phys. Rev. Lett. 105, 136805 (2010)

49. Wang, Y., Li, S. \& Yi, J. Electronic and magnetic properties of Co doped MoS2 monolayer. Sci. Rep. 6, 24153 (2016). 
50. Wang, B. Q. et al. Effects of Cr-doping on the photoluminescence and ferromagnetism at room temperature in $\mathrm{ZnO}$ nanomaterials prepared by soft chemistry route. Mater. Chem. Phys. 113, 103-106 (2009).

51. Yang, J. H. et al. A study of structural, optical and magnetic properties of $\mathrm{Zn}_{0.97-\mathrm{x}} \mathrm{Cu}_{\mathrm{x}} \mathrm{Cr}_{0.03} \mathrm{O}$ diluted magnetic semiconductors. J. Alloy. Compd. 509, 3672-3676 (2011).

52. Gonze, X. et al. ABINIT: first-principles approach to material and nanosystem properties. Comput. Phys. Commun. 180, 2582-2615 (2009).

53. Torrent, M. et al. Implementation of the projector augmented-wave method in the ABINIT code: application to the study of iron under pressure. Comput. Mater. Sci. 42, 337-351 (2008).

54. Grimme, S. Semiempirical GGA-type density functional constructed with a long-range dispersion correction. J. Comput. Chem. 27, 1787-1799 (2006).

55. Cheng, Y. C., Zhang, Q. Y. \& Schwingenschlogl, U. Valley polarization in magnetically doped single-layer transition-metal dichalcogenides. Phys. Rev. B 89, 155429 (2014).

\section{Acknowledgements}

This work was financially supported by the National Key Research and Development Program of China (No. 2016YFA0300103, 2017YFA0402800), National Natural Science Foundation of China (Grants No.11435012, U1632263, 11775225, 11604341, 51790491, and 21533007), and the Foundation for Innovative Research Groups of the National Natural Science Foundation of China (11621063). The authors would like to thank BSRF, SSRF and NSRL for the synchrotron beamtime.

\section{Author contributions}

S.Q.W., Z.S., and W.Y. conceived the experiments and supervised the project; H.D., P.G., H.T., W.H., and C.M. performed the measurements; H.D., L.C., and L.W. fabricated the devices; X. L., W.Zhao., and Y.Y. performed the magnetism measurements; C.W. performed the DFT calculations; Z.S., W.Y., L.S., W.Zhang., and S.Q.W. analyzed the results; and S.Q.W., Z.S., and W.Y. wrote the paper with comments from all authors.

\section{Additional information}

Supplementary Information accompanies this paper at https://doi.org/10.1038/s41467 019-09531-0.

Competing interests: The authors declare no competing interests.

Reprints and permission information is available online at http://npg.nature.com/ reprintsandpermissions/

Journal peer review information: Nature Communications thanks the anonymous reviewers for their contribution to the peer review of this work.

Publisher's note: Springer Nature remains neutral with regard to jurisdictional claims in published maps and institutional affiliations.

cc (i) Open Access This article is licensed under a Creative Commons Attribution 4.0 International License, which permits use, sharing, adaptation, distribution and reproduction in any medium or format, as long as you give appropriate credit to the original author(s) and the source, provide a link to the Creative Commons license, and indicate if changes were made. The images or other third party material in this article are included in the article's Creative Commons license, unless indicated otherwise in a credit line to the material. If material is not included in the article's Creative Commons license and your intended use is not permitted by statutory regulation or exceeds the permitted use, you will need to obtain permission directly from the copyright holder. To view a copy of this license, visit http://creativecommons.org/ licenses/by/4.0/.

(C) The Author(s) 2019 